\title{
Midline lumbar fusion using cortical bone trajectory screws. Preliminary report
}

\author{
Mateusz Bielecki, Przemysław Kunert, Marek Prokopienko, Arkadiusz Nowak, Tomasz Czernicki, Andrzej Marchel \\ Department of Neurosurgery, Medical University of Warsaw, Warsaw, Poland
}

Videosurgery Miniinv 2016; 11 (3): 156-163

DOI: 10.5114/wiitm.2016.62289

\begin{abstract}
Introduction: Midline lumbar fusion (MIDLF) using cortical bone trajectory is an alternative method of transpedicular spinal fusion for degenerative disease. The new entry points' location and screwdriving direction allow the approach-related morbidity to be reduced.

Aim: To present our preliminary experience with the MIDLF technique on the first 5 patients with lumbar degenerative disease and with follow-up of at least 6 months.

Material and methods: Retrospective analysis was performed on the first 5 patients with foraminal (4) or central (1) stenosis operated on between December 2014 and February 2015. Three patients were fused at L4-L5 and two at the L5-S1 level.

Results: No intra- or post-operative complications occurred with this approach. An improvement regarding the leading symptom in the early postoperative period (sciatica 4/4, claudication 1/1) was achieved in all patients. The mean improvements in the visual analogue scale for low back and leg pain were 2.2 and 4.8 respectively. The mean Oswestry Disability Index scores were 52\% (range: 16-82\%) before surgery and 33\% (range: 12-56\%) at 3-month follow-up (mean improvement 19\%). At the most recent follow-up, 4 patients reported the maintenance of the satisfactory result. The early standing and follow-up X-rays showed satisfactory screw placement in all patients.

Conclusions: In our initial experience, the MIDLF technique seems to be an encouraging alternative to traditional transpedicular trajectory screws when short level lumbar fusion is needed. Nevertheless, longer observations on larger groups of patients are needed to reliably evaluate the safety of the method and the sustainability of the results.
\end{abstract}

Key words: pedicle screw, midline lumbar fusion, cortical bone trajectory, minimally invasive spine surgery, lumbar degenerative disease.

\section{Introduction}

Midline lumbar fusion (MIDLF) using cortical bone trajectory (CBT) is an alternative method of transpedicular spinal fusion. The starting entry points located at the pars interarticularis are much closer to the midline than traditional entry points. Unlike the conventional method, MIDLF uses shorter and thinner screws, which are directed from inferomedial to superolateral, anchoring to a denser cortical layer of bone. Such a "reverse" screw trajectory and much more medial entry points make it possible to decrease the length of surgical incision and may reduce approach-related morbidity.

Experimental biomechanical studies indicate that CBT screws are of equivalent or even higher strength when compared to conventional transpedicular trajectory screws regarding pullout forces [1]. However, there is a paucity of evidence demonstrating the clinical effectiveness of this recently developed technique.

\section{Address for correspondence}

Przemysław Kunert MD, PhD, Department of Neurosurgery, Medical University of Warsaw, 1a Banacha St, 02-097 Warsaw, Poland,

phone: +48 606935 939, e-mail: pkunert@wp.pl 


\section{Aim}

To present our preliminary experience with the MIDLF technique on the first 5 patients with lumbar degenerative disease and with follow-up of at least 6 months.

\section{Material and methods}

\section{Patients and symptoms}

The first 5 patients with at least 6 months' follow-up after a one-level MIDLF procedure for lumbar degenerative disease were retrospectively analyzed. The group included 4 men and 1 woman ranging in age from 36 to 63 years old (mean 49 years old). All the procedures were performed between December 2014 and February 2015 by one neurosurgeon (PK).

In 4 patients, the main indication for surgery was foraminal stenosis, including 3 patients after prior microdiscectomies and 1 patient with L5-S1 retrolisthesis not operated on before. These 4 patients presented with chronic sciatica, and 3 of them had radicular leg weakness. In 1 case, the indication for surgery was the critical central and foraminal stenosis at L4-L5 due to degenerative disc disease and hypertrophic facets (Photo 1). The patient presented with claudication, low back pain (LBP) and minor sciatica. The details of the patients are shown in Table I. In all patients, the indication for interbody fusion was the need for, at least, unilateral facetectomy.

\section{Procedure}

Under general anesthesia and in the prone position, a midline incision of about $5-6 \mathrm{~cm}$ was made over the spinous processes at the affected level. The dorsolumbar fascia was incised bilaterally, preserving the supra- and interspinous ligaments. Gentle bilateral muscle dissection along the spinous process over the lamina was performed up to the lateral edge of the pars interarticularis and intervertebral joints. The usual lumbar retractors as used for microdiscectomy purposes were used. About $3 \mathrm{~mm}$ deep, four pilot holes at the starting points for CBT were marked by tapping the pars under lateral fluoroscopic guidance. The starting points, located on the pars interarticularis just medially to its lateral border and just caudally to the transverse process lower edge, reflected the medio-caudal footprint of the pedicle. The spinal canal and intervertebral foramina were

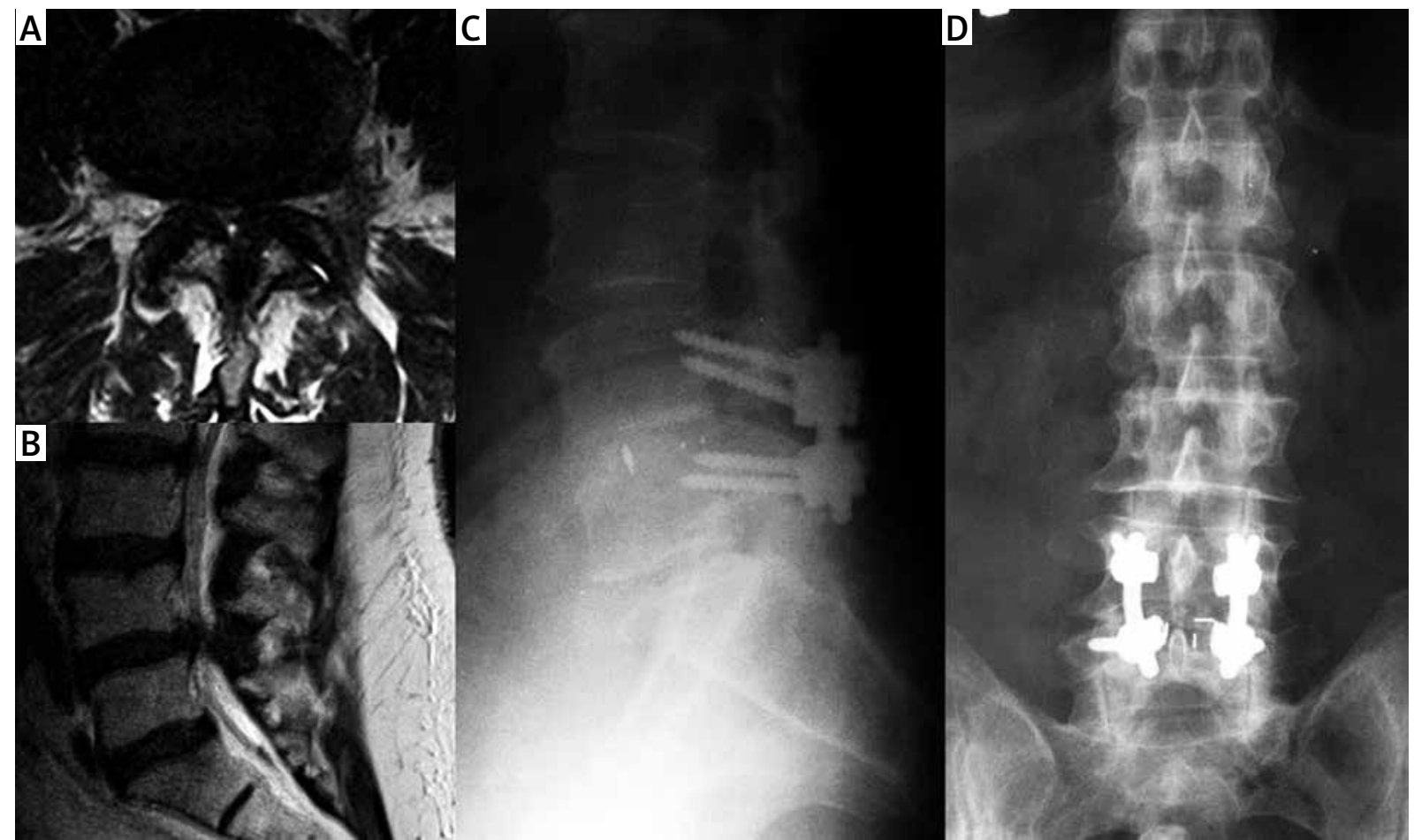

Photo 1. Preoperative MRI imaging (A, B) and follow-up radiographs (C, D) of the patient with L4-L5 central and foraminal stenosis, presented with claudication 


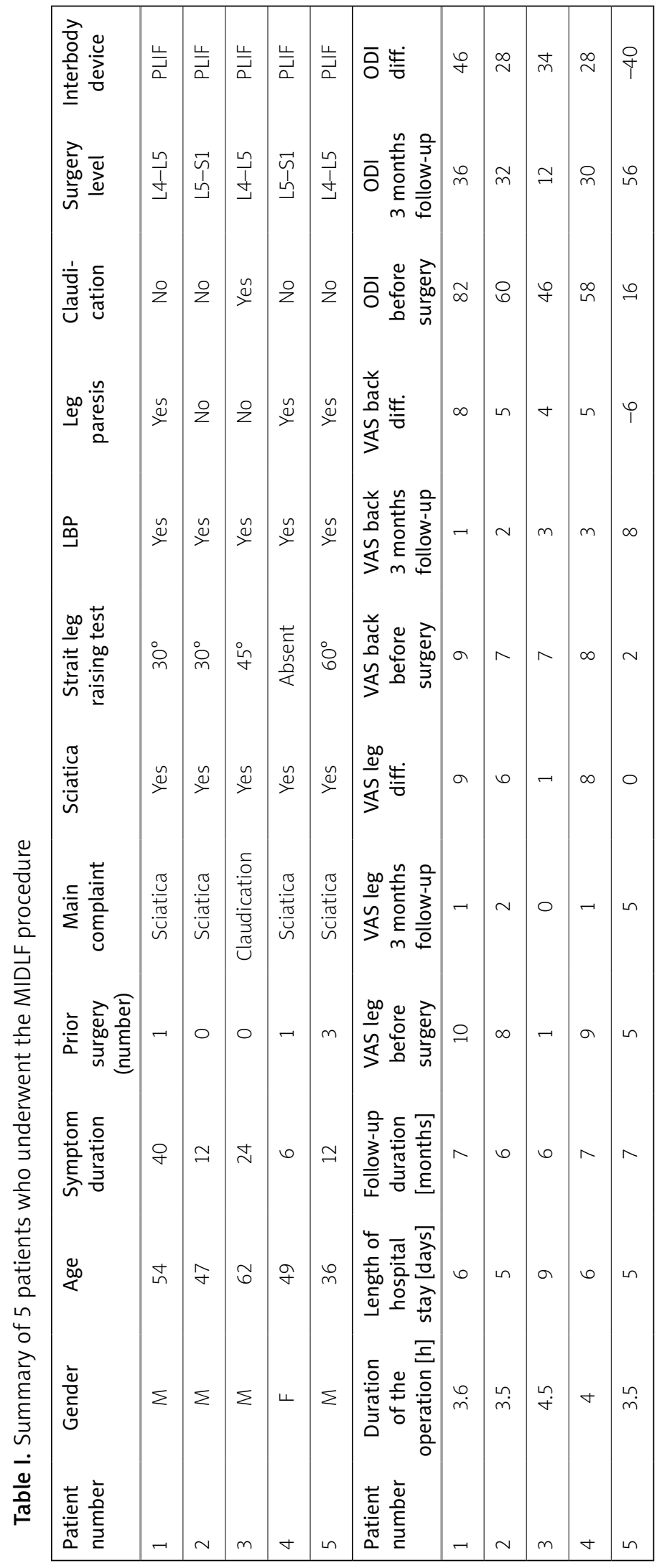


opened via bilateral laminotomy and facetectomy with sparing of the spinous process (Photo 2). Microscope magnification was utilized during dural sac and nerve root decompression. A bone margin of at least $3 \mathrm{~mm}$ around the starting points was left. After the intervertebral disc removal from both sides, the anterior part of the intervertebral space was densely filled with autograft (bone chips harvested during decompression). An interbody device (Capstone Peek, Medtronic Sofamor Danek, USA) was also placed in all 5 patients. The bone autograft with hydroxyapatite nanoparticle gel (Nanogel, Teknimed, France) was also placed into the central cavity of the device. Posterior lumbar interbody fusion (PLIF) technique was applied as a rule to achieve a symmetrical two-point interbody support. The posterior part of the interbody space was left empty to avoid bone chip migration toward the neural structures. Next, the screw channels were finished by tapping the same diameter holes as the planned screws. The entire screw length was tapped because of cortical bone hardness. The CBT was created with oblique, caudal-to-cephalad and medial-to-lateral direction under fluoroscopy control. After verifying the correctness of the channels with a probe, the cortical screws were placed. The diameter of the screws was $4.5 \mathrm{~mm}$ and their lengths ranged from $25 \mathrm{~mm}$ to $35 \mathrm{~mm}$ (Medtronic Sofamor Danek, USA). Care was taken to avoid injury to the upper joint capsule during the whole procedure. To avoid prolonged bilateral muscle retraction, both sides were dissected, decompressed and fused consecutively (with contralateral muscle relaxation). Wounds were closed in standard fashion, leaving drains if needed (4/5). Three patients were fused at $\mathrm{L} 4-\mathrm{L} 5$ and two at $\mathrm{L} 5-\mathrm{S} 1$.

\section{Outcome assessment}

The length of hospital stay and operative time were analyzed. The functional status was assessed using the Oswestry Disability Index (ODI). The pain intensity, separately for low back and sciatica, was evaluated using the visual analogue scale (VAS). Control standing radiograph of the lumbar spine was performed in all patients before discharge from hospital and a second time 3 months after surgery. The X-ray scans were evaluated and compared for signs of hardware failure, screw loosening and spinal instability. Follow-up computed tomography (CT) and magnetic resonance imaging (MRI) were

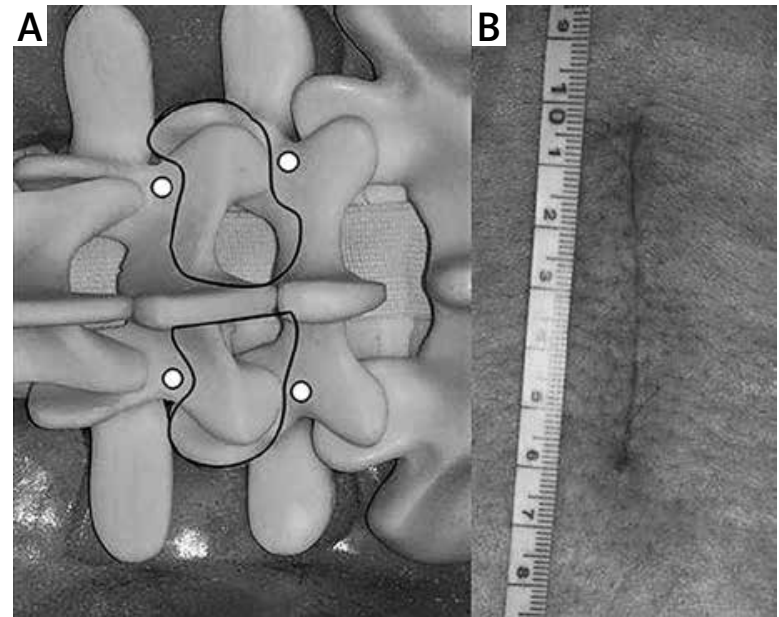

Photo 2. A - Extent of bony resection for decompression (black transparent outline): bilateral laminotomy and facetectomy with sparing of the spinous process. Starting points on pars interarticularis (white dots) are located just medially to its lateral border and just caudally to the transverse process lower edge. B - The scar after the MIDLF procedure

performed in case of ongoing significant pain. The outcome was evaluated three times: at discharge, 3 months after surgery (follow-up visit) and 6 months after surgery (a telephone conversation or email correspondence). The average length of follow-up was 6.6 months (range: 6-7 months).

\section{Results}

The length of hospital stay ranged from 5 to 9 days (mean: 6.2 days). The mean operating duration was $3.8 \mathrm{~h}$ (range: $3.5-4.5 \mathrm{~h}$ ). No intra- or postoperative complications occurred with this approach. No pedicle fractures at the insertion site were noted. No patient had surgery-related nerve root injury. Ambulation on postoperative day 1 was possible in all 5 patients. An improvement regarding the leading symptom in the early postoperative period (sciatica $4 / 4$, claudication 1/1) was also achieved in all patients. The early standing X-rays showed satisfactory CBT screw placement in all patients. In follow-up radiographs, there were no signs of screw loosening or other hardware failure such as angulation, fracture or disconnection.

During a follow-up visit at 3 months after surgery, 4 patients reported maintenance of the satisfactory result. One patient reported being dissatisfied with 
his clinical result, because of LBP and sciatica recurrence (Table I). This patient previously underwent unilateral microdiscectomy three times at the L4-L5 level with interspinous spacer implantation during the last revision. The MRI and CT imaging performed after the MIDLF procedure revealed adequate decompression of the neural structures and no signs of hardware failure (Photo 3). Follow-up X-ray showed a stable position of the screws compared to an earlier radiograph. The patient was referred to a pain specialist. Recently, he was offered spinal cord stimulation for ongoing back and leg pain, but he did not consent.
At the most recent follow-up, no patient was revised and no patient demonstrated evidence of screw loosening. Four patients still reported being satisfied with the result of surgery.

According to the VAS, the mean LBP intensity was as follows: before surgery 6.6 (range: 2-9), before discharge 4.8 (range: 0-9), and at 3-month follow-up 4.4 (range: 1-8). For leg pain, the mean VAS values were: before surgery 6.6 (range: 1-10), before discharge 3.0 (range: 1-5), and at 3-month follow-up 1.8 (range: $1-5)$. The mean improvements in VAS for LBP and leg pain were 2.2 and 4.8 respectively. The mean ODI scores were $52 \%$ (range:

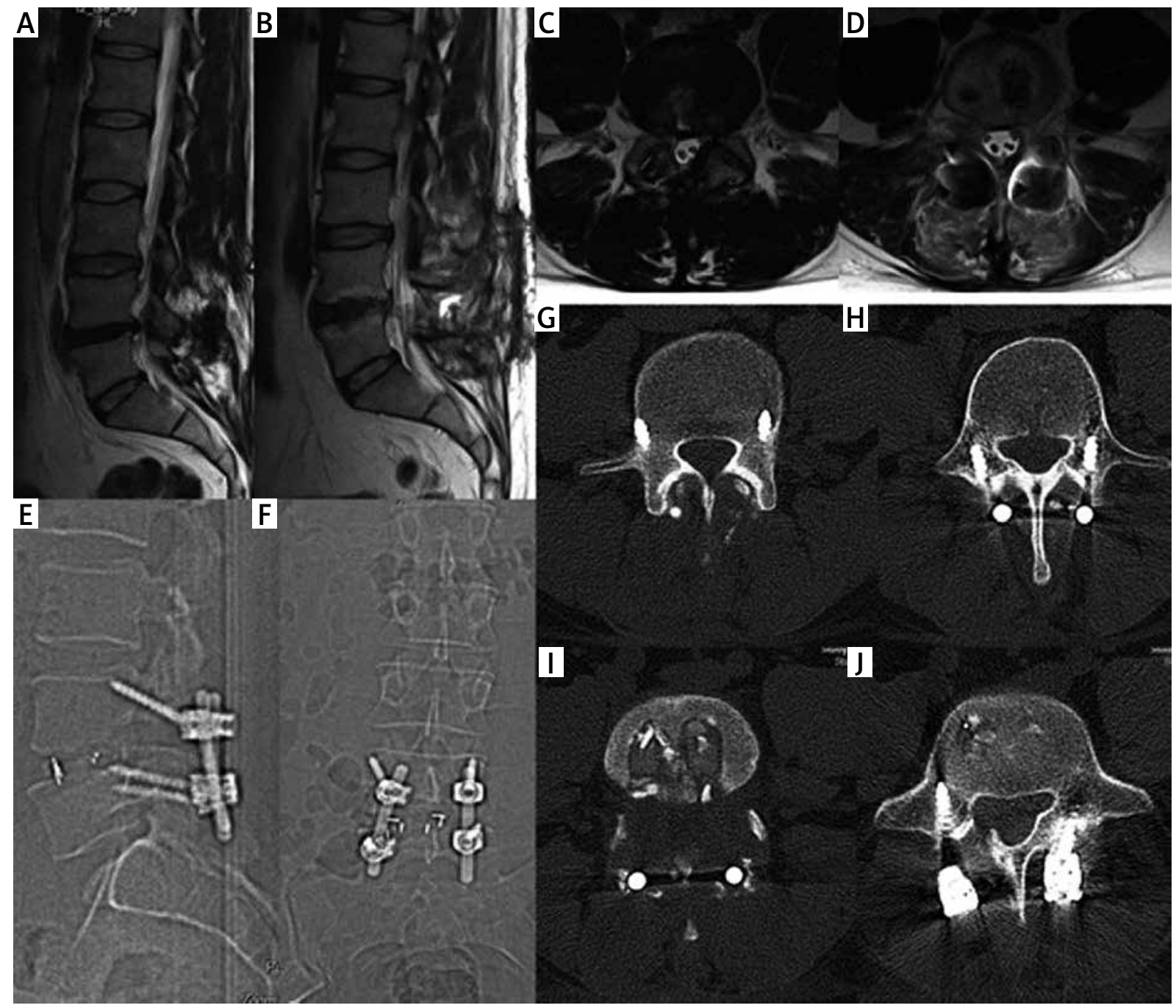

Photo 3. The MRI and CT imaging of the patient who was not satisfied with his surgery result. $\mathrm{A}, \mathrm{C}-$ preoperative MRI imaging showing L4-L5 foraminal stenosis. B, D - follow-up MRI revealing adequate decompression of the neural structures. E-J - Follow-up CT imaging demonstrating an appropriate cortically based trajectory of the screws and proper location of interbody devices 
16-82\%) before surgery and 33\% (range: $12-56 \%$ ) at 3-month follow-up. A mean improvement of $19 \%$ on the ODI compared with the preoperative baseline was achieved.

\section{Discussion}

In 2009, Santoni et al. introduced a new idea for lumbar pedicle screw placement called "cortical bone trajectory" [1]. Their cadaveric biomechanical study showed that CBT screws have higher resistance to uniaxial pullout forces than traditionally directed pedicle screws. Recent studies confirmed this finding and showed that the particular trajectory is probably more important than a specially designed screw thread for this purpose [2, 3]. However, CBT screws may not be so resistant to more physiological cyclic multidirectional loads and micromovements as the longer and thicker screws which are traditionally used [4]. Data from experimental studies are somewhat conflicting $[5,6]$.

The main advantage of the MIDLF technique is that the spinal canal and intervertebral foramina de- compression and also discectomy, interbody fusion and screw fixation are all possible with one limited midline incision and a familiar approach. Despite the screws being shorter and thinner, CBT provides greater adherence of the screw thread to higher density bone [7]. The risk of spinal canal violation by a screw is very low because of the medio-lateral trajectory direction. However, the theoretical advantages and promising results of biomechanical tests have to be confirmed in vivo. Unfortunately, there is a lack of long-term clinical observations evaluating the durability of results, and only a few clinical reports on short- and mid-term outcomes exist [4, 8-10].

The first prospective, randomized and non-inferiority trial comparing traditional and CBT screws in PLIF confirmed comparable fusion rates and clinical results for both techniques at 1-year follow-up. Nevertheless, comparisons of incision length, operative time and blood loss volume demonstrated the superiority of the MIDLF procedure [10]. In general, the range of minimally invasive spine surgery techniques has several proven advantages, including less blood loss, less need of transfusion, less postopera-
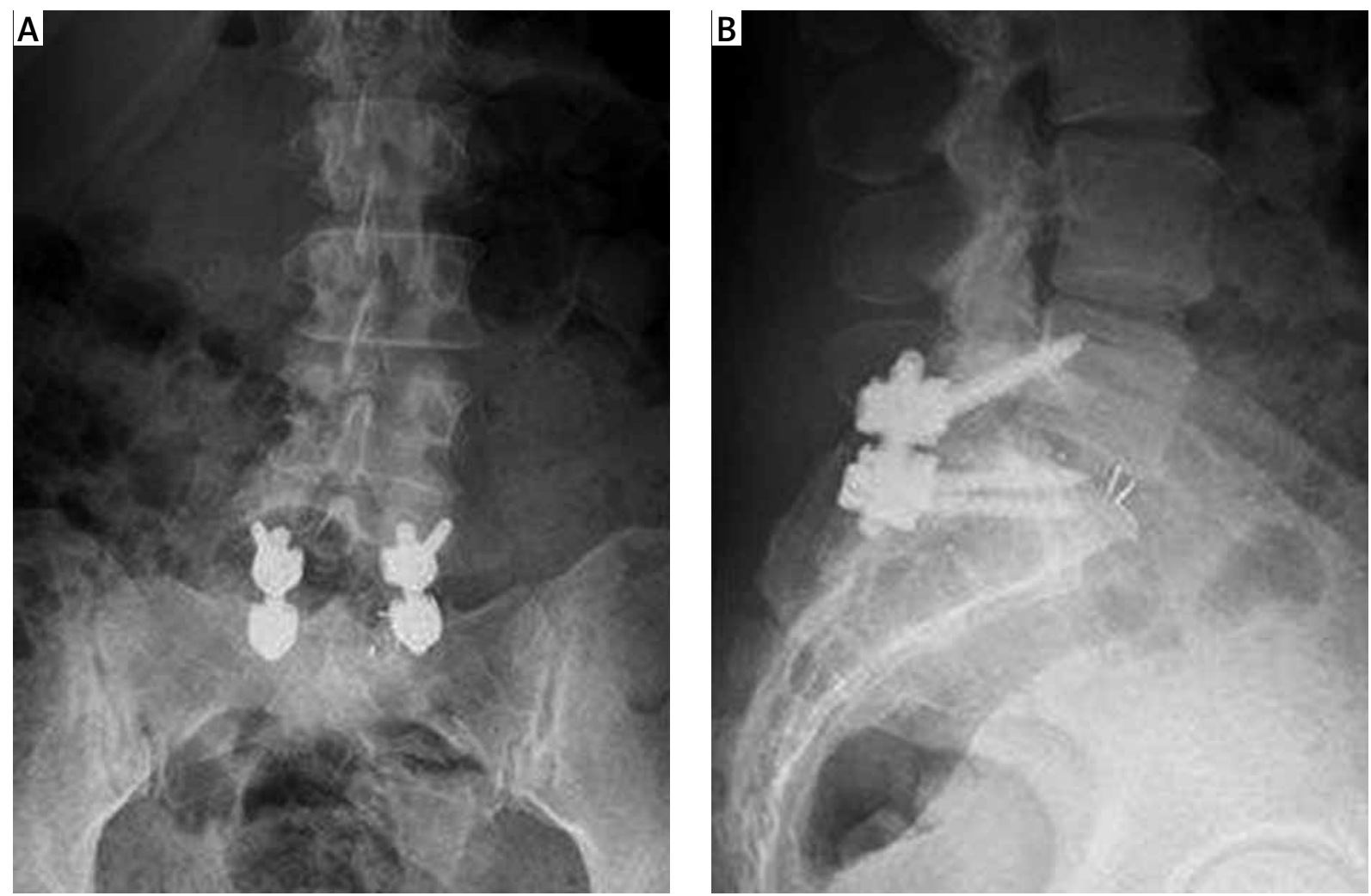

Photo 4. Standing postoperative radiographs demonstrating the penetration of one of the S1 screws into the L5-S1 disc space 
tive back pain, shorter recovery time to ambulation, and shorter length of hospital stay $[11,12]$.

Our initial series shows that good results with this new technique can be achieved from the very beginning. This early experience allows us to conclude that the procedure is relatively easy to learn as compared to traditional transpedicular screw placement. However, the operative time of $3.8 \mathrm{~h}$ reflects the difficulties with the introduction of a new technique. In the series of Kasukawa et al., the operative duration was $3.5 \mathrm{~h}$ on average [9]. In the cited paper, transforaminal lumbar interbody fusion (TLIF) procedures with 'conventional', percutaneous and CBT pedicle screw insertions were compared. Bone fusion rates, lordotic angle maintenance and correctness of pedicle screw position were comparable among these three techniques at follow-up. However, blood loss volume was smaller in the MIDLF technique [9].

We have read with interest the recent paper of Glennie et al. [4], who reported the loss of slip reduction less than 1 year after surgery in 4 of 8 patients operated on with the MIDLF technique. However, 3 of these 4 patients had no interbody device. Therefore, in accordance with the conclusions of the authors, we believe that MIDLF should be, as a rule, used in combination with an interbody fusion. Biomechanical flexibility tests showed that in the case of an intact disc, traditional pedicle screws deliver better protection during axial rotation than CBT screws [5]. However, there were no differences when direct lateral fusion (DLIF) interbody support was implanted. Also, the results obtained by Perez-Orribo et al. suggest that a symmetrical DLIF support may better cooperate with MIDLF than an asymmetrical TLIF [5]. For this reason, we decided to employ PLIF as a symmetrical support, which can be implanted via the same approach. Such a strategy refers to the idea that the screw-rod construct acts as a temporary internal brace, up to the time when interbody fusion will be efficient. However, the question whether MIDLF is strong enough for durable slip reduction remains to be answered. Recently, Mizuno et al. reported the use of the MIDLF technique in isthmic and degenerative spondylolisthesis in 12 patients with stable radiological results during a mean follow-up period of 15 months [8]. To date, we have used the MIDLF procedure when lumbar fusion was needed after foraminal nerve root decompression in cases without spondylolisthesis, and for this purpose CBT screws seem to be fully sufficient.
Technical notes. Initially, the lateral direction for a cortical screw placement seemed to be approximately $20^{\circ}$ medial-to-lateral. However, the morphometric study by Matsukawa et al. showed that the lateral angle is about 8.5 to $9^{\circ}$ for the L1-L5 levels [13]. Therefore, spinous process removal is not needed only for potential conflict with the trajectory. Consequently, in general, we try to preserve the spinous process with its ligaments as part of the minimally invasive surgery policy. In the case of L5S1 fusion, we adopted a "penetrating S1 endplate screw technique" (Photo 4) [14]. Such CBT at the S1 level is angulated cranially, similar to CBT at the L1-L5 levels, but the screw protrudes into the intervertebral disc space, providing stronger bicortical adherence.

\section{Conclusions}

In our initial experience, the MIDLF technique seems to be an encouraging alternative to traditional transpedicular trajectory screws when short level lumbar fusion is needed. Nevertheless, longer observations on larger groups of patients are needed to reliably evaluate the safety of the method and the sustainability of the results.

\section{Conflict of interest}

The authors declare no conflict of interest.

\section{References}

1. Santoni BG, Hynes RA, McGilvray KC, et al. Cortical bone trajectory for lumbar pedicle screws. Spine J 2009; 9: 366-73.

2. Ueno M, Sakai R, Tanaka K, et al. Should we use cortical bone screws for cortical bone trajectory? I Neurosurg Spine 2015; 22: 416-21.

3. Wray S, Mimran R, Vadapalli S, et al. Pedicle screw placement in the lumbar spine: effect of trajectory and screw design on acute biomechanical purchase. J Neurosurg Spine 2015; 22: 503-10.

4. Glennie RA, Dea N, Kwon BK, et al. Early clinical results with cortically based pedicle screw trajectory for fusion of the degenerative lumbar spine. J Clin Neuroscin 2015; 22: 972-5.

5. Perez-Orribo L, Kalb S, Reyes PM, et al. Biomechanics of lumbar cortical screw-rod fixation versus pedicle screw-rod fixation with and without interbody support. Spine 2013; 38: 635-41.

6. Baluch DA, Patel AA, Lullo B, et al. Effect of physiological loads on cortical and traditional pedicle screw fixation. Spine 2014; 39: 1297-330.

7. Kojima K, Asamoto S, Kobayashi Y, et al. Cortical bone trajectory and traditional trajectory - a radiological evaluation of screw-bone contact. Acta Neurochir (Wien) 2015; 157: 1173-8. 
8. Mizuno M, Kuraishi K, Umeda Y, et al. Midline lumbar fusion with cortical bone trajectory screw. Neurol Med Chir (Tokyo) 2014; 54: 716-21.

9. Kasukawa Y, Miyakoshi N, Hongo M, et al. Short-term results of transforaminal lumbar interbody fusion using pedicle screw with cortical bone trajectory compared with conventional trajectory. Asian Spine J 2015; 9: 440-8.

10. Lee GW, Son JH, Ahn MW, et al. The comparison of pedicle screw and cortical screw in posterior lumbar interbody fusion: a prospective randomized noninferiority trial. Spine J 2015; 15: 1519-26.

11. Park Y, Ha JW. Comparison of one-level posterior lumbar interbody fusion performed with a minimally invasive approach or a traditional open approach. Spine 2007; 32: 537-43.

12. Kubaszewski L, Kaczmarczyk J, Nowakowski A, et al. Foraminoplastic transfacet epidural endoscopic approach for removal of intraforaminal disc herniation at the L5-S1 level. Videosurgery Miniinv 2014; 9: 96-100.

13. Matsukawa K, Yato Y, Nemoto O, et al. Morphometric measurement of cortical bone trajectory for lumbar pedicle screw insertion using computed tomography. I Spinal Disord Tech 2013; 26: 248-53.

14. Matsukawa K, Yato Y, Kato T, et al. Cortical bone trajectory for lumbosacral fixation: penetrating S-1 endplate screw technique: technical note. J Neurosurg Spine 2014; 21: 203-9.

Received: 10.01.2016, accepted: 5.09.2016 